\title{
Heterogeneity of Cell Death
}

\author{
J.B. Stevens ${ }^{a}$ B.Y. Abdallah ${ }^{a} \quad$ G. Liu ${ }^{a} \quad$ S.D. Horne ${ }^{a} \quad$ S.W. Bremer ${ }^{a} \quad$ K.J. Ye \\ J.Y. Huang ${ }^{a}$ M. Kurkinen ${ }^{a}$ C.J. Ye ${ }^{b}$ H.H.Q. Heng ${ }^{a, c, d}$
}

${ }^{a}$ Center for Molecular Medicine and Genetics, and Departments of ${ }^{b}$ Internal Medicine and ${ }^{c}$ Pathology, Wayne State

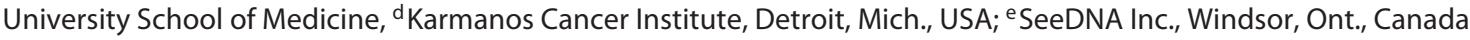

\section{Key Words}

Cancer evolution - Cell death heterogeneity $\cdot$ Chromosome fragmentation - Genome chaos · Genome theory · Mitotic cell death $\cdot$ Non-clonal chromosomal aberration

\begin{abstract}
Cell death constitutes a number of heterogeneous processes. Despite the dynamic nature of cell death, studies of cell death have primarily focused on apoptosis, and cell death has often been viewed as static events occurring in linear pathways. In this article we review cell death heterogeneity with specific focus on 4 aspects of cell death: the type of cell death; how it is induced; its mechanism(s); the results of cell death, and the implications of cell death heterogeneity for both basic and clinical research. This specifically reveals that cell death occurs in multiple overlapping forms that simultaneously occur within a population. Network and pathway heterogeneity in cell death is also discussed. Failure to integrate cell death heterogeneity within analyses can lead to inaccurate predictions of the amount of cell death that takes place in a tumor. Similarly, many molecular methods employed in cell death studies homogenize a population removing heterogeneity between individual cells and can be deceiving. Finally, and most importantly, cell death heterogeneity is linked to the formation of new genome systems through induction of aneuploidy and genome chaos (rapid genome reorganization).
\end{abstract}

Copyright $\odot 2013$ S. Karger AG, Basel

\section{KARGER}

E-Mail karger@karger.com

www.karger.com/cgr
Cell death is a diverse and complex group of processes occurring in a number of forms that is ultimately caused by stress to the cellular system [Stevens et al., 2011a; Galluzzi et al., 2012]. Of these types of cell death, apoptosis is the most studied, as it has been the primary focus of cell death research for decades. Measures of apoptosis, however, are inadequate at estimating the amount of cell death that occurs [Hendry and West, 1997]. For example, in cancer, apoptosis does not always faithfully represent the amount of cell death occurring in a tumor [Kondo, 1995; Erenpreisa and Cragg, 2001; Abend, 2003]. It has been suggested that mitotic cell death (MCD) represents the major form of cell death that occurs in treated tumors with autophagy and necrosis also playing a role in the reduction of tumor mass [Heng et al., 2004; Stevens et al., 2004, 2007, 2011a]. Thus, more than one type of cell death occurs in the same tissue at the same time.

Cells within a population exhibit heterogeneous behaviors especially in disease conditions, and cell death is not a unique property in this regard [Heppner, 1984; Hendry and West, 1997; Davidoff, 2009; Heng et al., 2009; Stevens et al., 2011b; Heng, 2013b]. This heterogeneity occurs at both biochemical and morphological levels. For instance, similar cell death morphologies can be produced by different biochemical pathways [de Bruin and Medema, 2008; Galluzzi et al., 2012]. The type(s) of cell death that occur rely on a complex interplay between the factors that induce the cell death, cellular status (such as 
genome stability) and the availability of proteins, complexes, organelles, and other factors required for a given cell death [Hendry and West, 1997; Calzone et al., 2010; Stevens et al., 2011a]. Cell death also does not occur at the same rate in individual cells [Goldstein et al., 2000a, b; Tyas et al., 2000]. Thus, cell death can be unpredictable at times (e.g. during chemotherapy), while at other times it occurs in a predictable manner (e.g. during Caenorhabditis elegans development) [Lettre and Hengartner, 2006; Domingos and Steller, 2007]. On top of this, heterogeneity occurs within normal cellular populations and populations in diseased tissue exhibit increased heterogeneity. For instance, genetic/genomic heterogeneity is ubiquitous in cancer [Heng, 2007; Heng et al., 2011a]. This genetic heterogeneity enhances functional heterogeneity of cells in a population.

In addition to cell death occurring in multiple forms, the outcomes of cell death can also be heterogeneous [Erenpreisa and Cragg, 2001; Stevens et al., 2011a]. Cell death does not always occur with complete fidelity. For a multitude of reasons the execution of cell death can be interfered with, leading to cells with varying degrees of damage, including DNA damage. This damage then can become fixed in the form of mutations, copy number variations or gross chromosomal alterations which can be inherited by progeny. Not surprisingly, escape from cell death due to drug treatment is directly attributable to population variability in protein expression, not a specific pattern of expression in cells [Cohen et al., 2008]. Cell death heterogeneity lies at the heart of one of the key challenges for cancer therapy, drug resistance [Heng et al., 2010a; Stevens et al., 2011a]. Cells that are resistant to the form(s) of cell death induced by a specific drug can quickly become the dominant cell type in a tumor if all other cells are effectively eliminated. Furthermore, the stress of drug treatment can spur adaptive genome evolution such as genome chaos, increasing the likelihood for survivors [Heng et al., 2006b, 2011a, b]. Thus not all cell death-related processes result in a favorable outcome in treating cancer. Increasing our understanding of cell death processes and their inherent heterogeneity will improve our ability to systematically treat multiple diseases, especially cancer.

\section{Multiple Types of Cell Death}

In order to fully appreciate the heterogeneity of cell death, some background on the various forms of cell death is required.

Heterogeneity of Cell Death

\section{Apoptosis}

Apoptosis has been the traditional focus of much of the cell death field since its discovery in 1972 [Kerr et al., 1972]. During this time apoptosis has been shown to be a complex process that can occur via multiple pathways in response to multiple stimuli. Prototypical apoptosis occurs in a mitochondrial-dependent manner through activation of caspase proteases which form a cascade and ultimately result in the degradation of nuclear material. Cellular contents are then packaged into blebs that can then be taken up and destroyed by neighboring cells. However, a multitude of apoptotic pathways have been uncovered including intrinsic pathways that are responsive to signals within the cell such as DNA damage or reactive oxygen species (ROS) exposure and extrinsic pathways instituted by cell-cell interactions. Furthermore, apoptosis occurs both in caspase-dependent and independent manners and similarly can be dependent or not on mitochondrial function [Galluzzi et al., 2012].

\section{Necrosis}

Necrosis occurs both as an unregulated and a regulated form of cell death due to overwhelming stress including drug toxicity, ROS exposure or mechanical damage or as a regulated form of cell death upon certain conditions. Regulated necrosis can occur in response to a number of factors, especially if the caspase system is compromised. The RIP family of proteins is often used as a marker of regulated necrosis [Galluzzi et al., 2009; Vandenabeele et al., 2010].

\section{Autophagy}

Autophagy is the process of self digestion which usually involves extensive vacuolization of the cytoplasm of the cell [Galluzzi et al., 2008b]. During the process, contents of the cell are isolated into phagosomes which fuse with lysosomes to form autophagosomes and digest the contents therein. Autophagy is primarily used to recycle organelles and large protein complexes and serves as a central system for adaptation to stress [Galluzzi et al., 2008b]; however, large scale autophagy can lead to the elimination of entire cells [Eisenberg-Lerner and Kimchi, 2009]. Like apoptosis, reduction of the ability to undergo autophagy increases the risk of cancer [Qu et al., 2003].

\section{Mitotic Cell Death}

Mitotic cell death (MCD) is the most common cell death occurring in tumors [Roninson et al., 2001]. MCD has been largely described on a morphological basis, though some progress has been made in identifying cel- 
lular and molecular mechanisms of MCD. Cells undergoing MCD often show nuclear aberrations such as multiand micronuclei. MCD occurs in 2 major forms, one occurring directly during mitosis (chromosome fragmentation or C-Frag), and one following a failed mitosis (mitotic catastrophe). Each of these types of MCD typically shares a prolonged mitotic arrest.

Mitotic catastrophe is a form of MCD that primarily occurs after a failed mitosis and involves some of the apoptotic machinery including caspase 2 activation [Castedo et al., 2004b]. TP53 and other TP53 family members have also been implicated with mitotic catastrophe in some cases [Nitta et al., 2004; Taylor et al., 2006]. TP53 and family members have been shown to impact mTOR (mammalian target of rapamycin) signaling and elicit senescence [Roninson et al., 2001].

C-Frag is another form of MCD that occurs directly during mitosis (fig. 1) [Stevens et al., 2004, 2007; Ye et al. 2007]. It is detectable in cytogenetic preparations, and it is identifiable by its unique morphology of degraded, condensed chromosomes and loss of cell membrane integrity [Heng et al., 2004; Stevens et al., 2007]. C-Frag is distinct from caspase-reliant apoptosis as it is not inhibited by broad spectrum caspase inhibitors or by overexpression of Bcl-2. PARP is also rapidly degraded during C-Frag, separating it from some forms of regulated necrosis [Stevens et al., 2011a]. In the past, C-Frag has been confused with premature chromosome condensation; however, these 2 phenomena are distinct [Stevens et al., 2007, 2010].

\section{Many Types of Cell Death Coexist}

Each type of cell death is not exclusive, different types of cell deaths often occur within the same cellular population [Abend, 2003]. Apoptosis and necrosis are often shown to occur as variable responses in a cell population to certain treatments [Xiao et al., 2002]. Similarly, apoptosis and autophagy can occur within the same population [Gonzalez Polo et al., 2005]. It has been argued whether these cell deaths are complementary or in opposition to each other. Autophagy (though not necessarily death by autophagy) occurs in response to stresses such as starvation and has been proposed to be a method of cellular adaptation. Autophagic death, however, can be induced or can occur if defects exist in apoptotic pathways [Galluzzi et al., 2008b, 2012]. It is therefore reasonable that co-occurrence of autophagy and apoptosis is both adaptive and a method to eliminate cells. C-Frag and apoptosis occur within the same populations of cancer cells with higher drug concentrations favoring apoptosis [Stevens et al.,

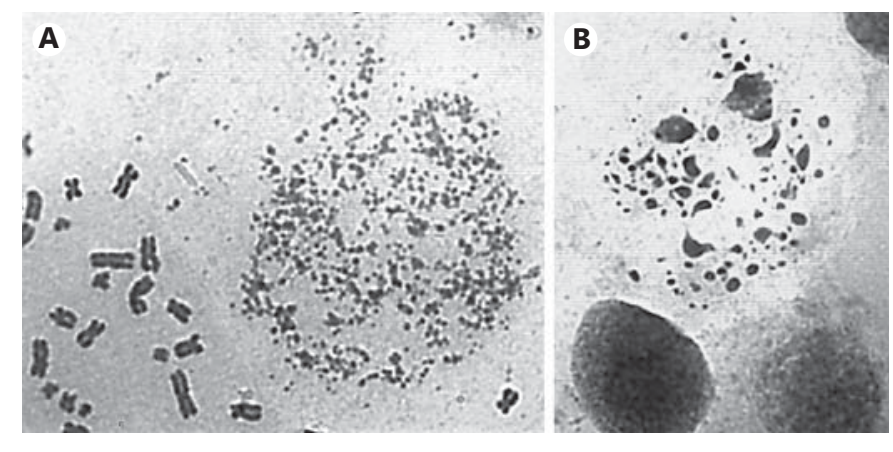

Fig. 1. Nuclear morphologies of cells undergoing C-Frag (A) and apoptosis (B). A During C-Frag, mitotic chromosomes are degraded (right part of photo). This is in contrast to normal chromosome morphology (left part of photo). B In apoptotic cells, uncondensed chromatin is degraded, forming condensed clusters of nuclear material (top) which are distinct from normal interphase nuclei (bottom).

2011a]. Mitotic catastrophe has been suggested to be a subtype of apoptosis resulting from a failed apoptosis, again suggesting that both forms of cell death are likely pervasive at the same time in response to the same treatment [Castedo et al., 2004a, b; Park et al., 2005, 2007; Galluzzi and Kroemer, 2009]. Thus, overlap of multiple types of cell death is an important issue. Studies based on identification of only one type of cell death are likely to underestimate the amount of cell death taking place, leading to confusion and unreliable treatment expectations.

\section{Heterogeneity of Factors That Induce Death}

Cell death can be induced by multiple factors, including changes in local morphogens, hormonal change, signals from neighboring cells, mechanical damage, or drug treatment [Entezari et al., 2010]. Apoptosis occurs as an extrinsic and intrinsic process [Galluzzi et al., 2012]. In extrinsic apoptosis, a number of molecules bind or are removed from receptors. These receptors include FAS, tumor necrosis factor receptor, netrin receptors and others [Galluzzi et al., 2012]. Intrinsic apoptosis is induced by multiple stresses, including many chemotherapeutics and ionizing radiation. Intrinsic apoptosis typically proceeds following mitochondrial outer membrane permeabilization, release of cytochrome $c$, and activation of apoptosis inducing factor, though it can occur independent of mitochondrial outer membrane permeabilization such as in the case of Bcl-2 or Mcl overexpression [Daugas et al., 2000; Sun et al., 2001; Riedl and Shi, 2004]. Au- 
tophagy typically occurs in response to low nutrient abundance as a survival mechanism but can also be induced by various drugs. MCDs also occur in response to various types of stress, often when cell cycle checkpoint function is abrogated, though they can also occur in normal tissue [Stevens et al., 2011a]. C-Frag, for instance, has been shown to occur in swine lymphocytes in response to vaccination [Nichols and Levan, 1965]. It also occurs in response to chemotherapeutic treatments, gene defects, ER stress, HSP90 inhibition, genomic instability and centrosome amplification. Taken together, cell death can be broadly viewed as a response to stress to which cells are unable to adapt [Stevens et al., 2011a].

\section{Heterogeneity of the Cell Death Process}

\section{Gene Level}

Gene expression in individual cells can be quite heterogeneous [Tantos et al., 2012], and resultant protein levels are also heterogeneous [Sigal et al., 2006]. Expression heterogeneity can be influenced by a multitude of factors, including cell cycle stage, transcriptional activity, differences in signaling in each cell, cell to cell communication, unequal separation of cellular contents during division, mutation, and genomic instability [Cohen et al., 2008]. Among these, cell cycle stage contributes approximately $20 \%$ of overall protein expression variability [Cohen et al., 2008]. Expression heterogeneity affects the ability of a cell to use specific pathways. For instance, if apoptotic pathways are inhibited by the lack of caspase expression, alternate cell death pathways and modalities are used. Furthermore, expression of cell death initiating and inhibiting proteins has been shown to occur in response to drug treatment in multimodal patterns; however, even though precise measurements of expression were made over a long time period, most expression changes did not predict outcome [Cohen et al., 2008].

Not only does gene level heterogeneity occur at the expression level, it also occurs at the functional level. Posttranslational modifications such as phosphorylation affect the activity and the interaction partners of a given protein, resulting in the propagation of a signal transduction cascade [Baek, 2011]. Other post-translational modifications affect the ability of cells to die. For instance, activating cleavage of some caspases releases inhibitors of apoptosis which in turn can inhibit caspase activity [Darding and Meier, 2012]. Furthermore, the evolutionary conservation of genes results in genes serving more than one function. Caspase 8 may be one such gene. It is well accepted that caspase 8 functions in the extrinsic apoptosis pathway, but conversely caspase 8 induces proliferation and is required for NF- $\mathrm{KB}$ activation in lymphocytes [Kennedy et al., 1999; Blagosklonny, 2003; Su et al., 2005]. TP53 is another well-known player in forms of cell death that also has many other functions, including transcriptional regulation [Feng et al., 2008; Olivier et al., 2009].

\section{Pathway Level}

The gene level heterogeneity discussed above ultimately has its effects on the pathways that function in a cell. For example, the pathways that function in a given cell are not static [Tantos et al., 2012]. These pathways turn on and off, and genes that function in one pathway can have different functions within other pathways. In regards to cell death this means that cells of the same type are capable of utilizing different pathways and undergoing different types of cell death. Furthermore, different networks function in different cell types, often making use of a protein differently than other cell types. Again, different cell types use different pathways and cell death routines to undergo death. The pathway or type of death is largely dependent on the networks that govern the function of a given cell type. Lastly, discussion of pathway level heterogeneity within the context of cell death heterogeneity is prudent. For the convenience of discussion, these types of pathway heterogeneity are listed below. These 3 mechanisms ease the categorization of pathway level heterogeneity, but it should be noted that there can be substantial overlap of these mechanisms. For instance, genome level heterogeneity can contribute to both intrinsic and extrinsic cell death heterogeneity.

Intrinsic Pathway Heterogeneity

Cell death pathway heterogeneity occurs in response to intrinsic factors; for instance, apoptosis can occur in response to intrinsic stimuli such as ROS production, ER stress or genome damage. Heterogeneity plays a role as the mitochondria may or may not be involved, and caspase activity may or may not play a role. When the function of one of these proteins or its related pathways is compromised, other cell death-related pathways can activate, and alternate forms of apoptosis or other cell death types occur.

\section{Extrinsic Pathway Heterogeneity}

Extrinsic pathways of apoptosis include signaling pathways that involve Fas and the Fas ligand, the TNF family, and the netrin receptors whose mechanisms are 
also heterogeneous. For example, binding of Fas or the TNF family by their ligands results in activation of caspase 8 through recruitment of proteins to the death-inducing signaling complex and the induction of apoptosis [Fuentes-Prior and Salvesen, 2004]. However, removal of netrin induces apoptosis through distinct mechanisms [Mille et al., 2009; Guenebeaud et al., 2010]. Thus, the microenvironment of a cell has an impact on whether or not it dies.

\section{Pathway Heterogeneity Due to Genome Change}

Genome level change in cancer is often unique to each cell within a tumor, and genome level alterations are observed even in normal tissues. The population heterogeneity that arises from this genome level change is extensive. The networks that these pathways form are emergent properties, dependent on the specific genome system under which they are organized [Heng, 2009, 2013a, b; Heng et al., 2011a]. In regards to cell death, these changes are expected to impact the cellular system in 2 ways. First, by changing the patterns of genes that are expressed, genome change alters how pathways function. Cell death pathways are not exempt from this. Like other pathways, cell death pathways are far from static in cells with altered genomes, and it is of little surprise that cells within a given heterogeneous population respond in a heterogeneous manner to cell death stimuli. Second, the complexity of apoptotic pathways is astonishing, especially considering that apoptosis is by far the most well characterized form of cell death. It is likely that other forms of cell death show similar complexity.

\section{Genome Level}

The functional heterogeneity of genes that play roles in cell death is noteworthy in most normal tissues where healthy cells contain an unchanged genome. This heterogeneity increases in cancer [Heng et al., 2009; Stevens et al., 2011b]. Mutations and genome level alterations such as translocations and aneuploidy occur universally in cancer and impact the heterogeneity of cell death. Mutations occur in a stochastic manner and are often not shared by cells from the same tumor [Bielas and Loeb, 2005]. The observable effects of mutations on cell death are primarily inhibition of cell death, as mutations that induce cell death would most likely not be observable. Genome level change has even greater impact on the heterogeneity of cell death in tumors. The creation of fusion proteins by chromosomal translocations is one such event that has gained notoriety. Fusion proteins have altered function such as the $B C R-A B L$ translocation in chronic myelogenous leukemia which results in a constitutively active ABL kinase that drives proliferation. Other fusion events have been associated with increased proliferation in prostate cancer [Horne et al., 2013a]. Genome level change does not, however, only affect cell death heterogeneity by creating abnormal fusion proteins. Genome level change affects cell death in that it changes expression through changes in copy number and higher level chromatin organization [Heng, 2013a]. One altered chromosome can affect expression levels of tens or hundreds of genes greatly affecting the pathways that a cell can utilize for processes such as death. Thus genome alteration creates a new system reliant on novel pathways. Most tumors are highly heterogeneous at the genome level which in turn promotes cell death heterogeneity within a single tumor [Heng et al., 2010b, 2011b; Stevens et al., 2011a].

\section{Heterogeneity Resulting from Cell Death}

The types of cell death that occur, the inputs that lead to it, the genes that contribute to it, and the pathways that control cell death are all heterogeneous, so it should come as no surprise that the results of cell death are also heterogeneous. Typically cell death routinely results in the elimination of a cell; however, cell death processes can be arrested, resulting in an incomplete cell death. Here we will discuss cell death heterogeneity in incomplete and complete cases of cell death.

\section{Incomplete Cell Death}

Incomplete cell death is important in a number of physiological processes. For example, caspase 8 activation is required for lymphocyte activation [Su et al., 2005]. Incomplete cell death carried out through activation of caspase 3 is also required for production of granulocytes and megakaryocytes [Galluzzi et al., 2008a]. Other instances of incomplete cell death, however, are not so regulated and create extensive genomic change that can drive cancer progression [Heng et al., 2006a, b, 2011b]. It has been hypothesized that incomplete apoptosis could lead to extensive genome change which may be responsible for a process called genome or karyotypic chaos and recently referred to as chromothripsis where one or more chromosomes have extensive complex translocations (fig. 2) [Heng et al., 2006b; Stephens et al., 2011]. It has been hypothesized that the chromosomal fragments that compose the derivative chromosomes in genome chaos/chromothripsis must stem from chromosomal breaks in mi- 

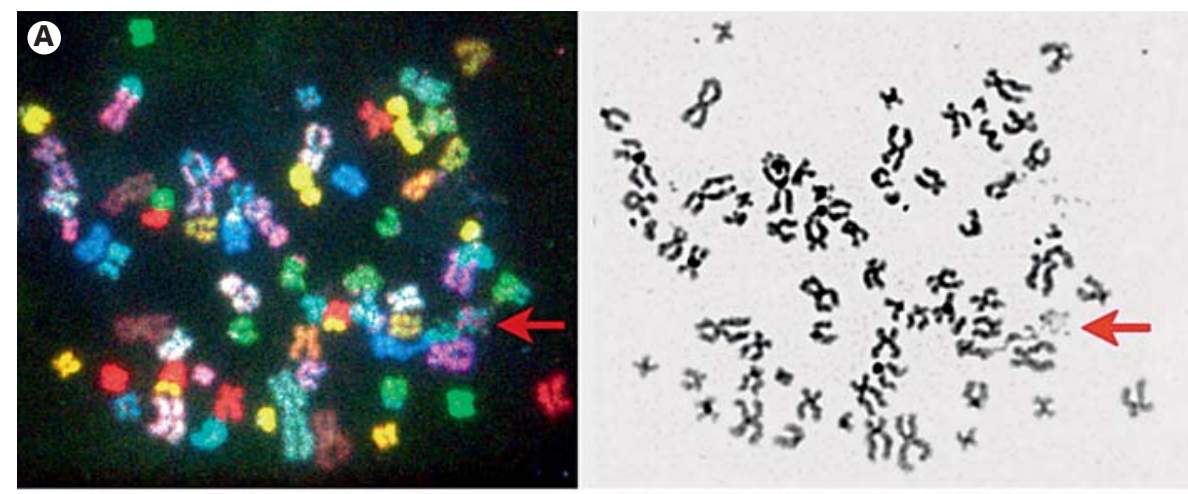

Fig. 2. Incomplete cell death can result in altered genomes. In both panels spectral karyotype images (SKY) are provided on the left, and reverse DAPI images are provided on the right. A Incomplete C-Frag can result in the degradation of one or more chromosomes, resulting in aneuploidy. In this figure, the chromosome being degraded is marked by a red arrow in both SKY and DAPI images. Reproduced from Stevens et al. [2007] with permission from Cancer Research. B Incomplete C-Frag can result in genome chaos when fragments of chromosomes recombine to form highly changed derivative chromosomes (red arrows).
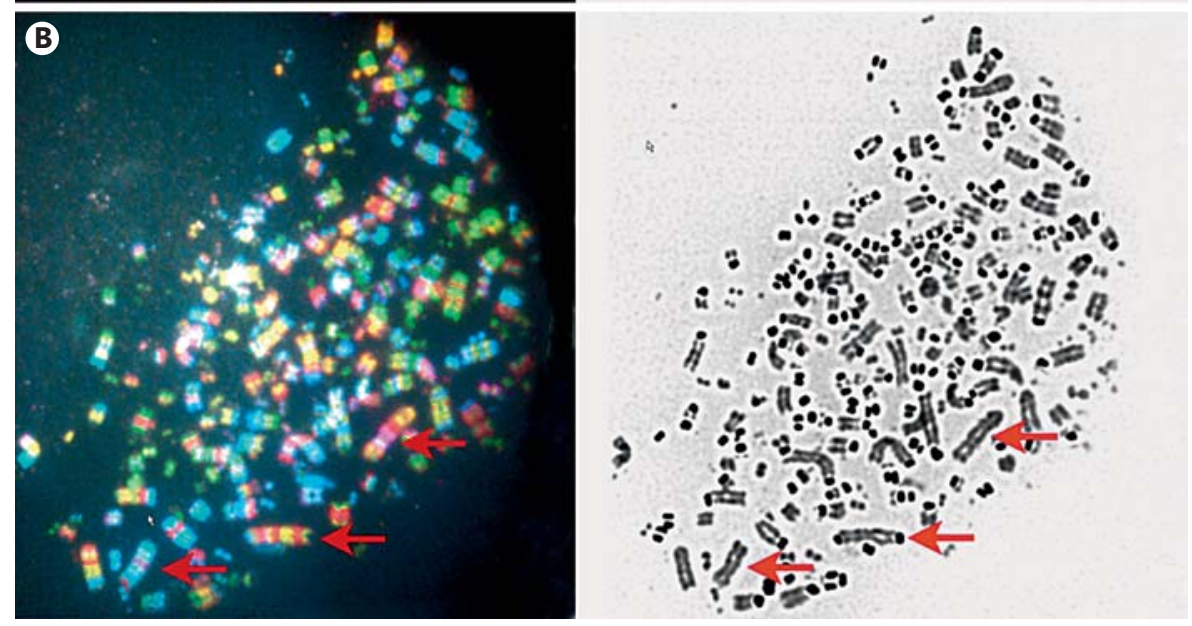

totic cells [Stevens et al., 2007, 2011a; Heng et al., 2011b; Stephens et al., 2011].

Our preliminary data shows that incomplete C-Frag followed by random rejoining of the fragments leads directly to genome chaos (fig. 2) [Liu et al., submitted]. In incomplete C-Frag, chromosomal fragments that are multiple megabases in size are rejoined apparently randomly. This leads to the production of amalgamated chromosomes composed of multiple fragments from 2 or more parental chromosomes. Incomplete C-Frag and autophagy can also degrade a single chromosome which in turn leads to aneuploidy [Sit et al., 1996; Stevens et al., 2007]. Thus, induction of cell death can in some cases lead to the alteration of a targeted cell, increasing the evolutionary potential of that cell and the probability that eventually a resistant cell will come about.

\section{Completed Cell Death}

Problems resulting from inappropriate cell death also arise from completed cell death. Most molecular research focuses on the process of cell death, but how the cell dies is not the only important part of the cell death story. While the cell death process is a response to stress, it can also cause new stress to the system. For example, the release of cellular contents upon necrotic cell death elicits an immune response which can damage and destroy neighboring cells. This immune reaction involves release of various ROS species into the extracellular space which can induce mutations and genome change, which ultimately introduces heterogeneity into the surrounding cells. In contrast to necrosis, during apoptosis cellular contents are packaged into blebs surrounded by cellular membrane to facilitate uptake by neighboring cells and avoid an immune response. While apoptosis does negate an immune response, it has also been shown to facilitate horizontal transfer of DNA that is packaged into the blebs [Bergsmedh et al., 2001]. This can result in genome change or copy number changes which are especially deleterious if these might be powerful oncogenes. Furthermore, for apoptotic cells to escape immune activation, apoptotic cells must be engulfed by neighboring cells or macrophages where they can be digested. Disruption to the engulfment of apoptotic cells has been implicated in autoimmune diseases such as lupus and arthritis [Nagata et al., 2010]. 


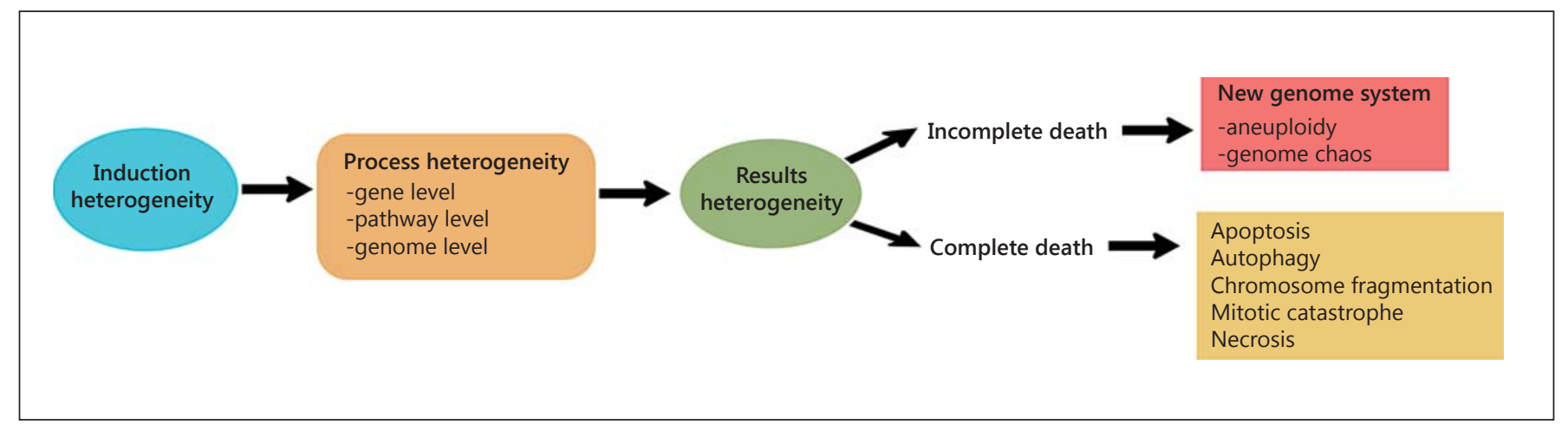

Fig. 3. Proposed model of the relationship between heterogeneity and various types of cell death and its potential impact on genome variation. Cell death heterogeneity as viewed from 3 key levels including heterogeneity of induction of cell death, processes and outcomes. Cell death heterogeneity is linked with different types of cell death and also serves as a source of genome alteration that is necessary material for somatic macro-evolution.

\section{Implications of Cell Death Heterogeneity}

Much of the current work in the cell death field is based on reductionist concepts and approaches. These concepts have been central to the development of our current understanding of cell death. The field has successfully linked many genes to various cell death pathways. It is true that each of these pathways can lead to cell death; however, the clinical utility of the continued identification of genes associated with cell death is limited when multiple types of cell death occur and a large number of pathways exist for each type of cell death. Thus, if nearly every pathway within a cell can be linked to cell death, what is the value of one isolated pathway, and how can switches in the pathways that are activated for cell death be predicted? In order to truly make an advance in cell death research, we must augment the currently known pathways and responses with the true complexity of living systems. To do so, multiple mathematical models are often applied to a biological function such as cell death. Multiple modeling techniques have been applied to increase understanding of apoptosis [Spencer and Sorger, 2011]. These models include ordinary differential equations, stochastic models, Boolean models, stability analysis, among others [Chen et al., 2007, 2010; Luan et al., 2007; Calzone et al., 2010]. An emerging concept to deal with this complexity is to study the evolutionary mechanism of a biological process rather than cataloging each minute molecular detail involved in that process. Studies of the overall impact of stress from a more systematic point of view may allow us to identify differences in system behavior(s) that better reflect changes in the overall state of the system. For example, we have shown that the evolutionary mechanism of cancer can be described as the sum of all molecular mechanisms and that the evolutionary potential of cancer is measured by genomic instability at the chromosome level [Heng, 2009; Heng et al., $2010 \mathrm{~b}, 2011 \mathrm{~b}$ ]. It was subsequently shown that focus on total genome change rather than specific gene alterations is a better predictor of cancer progression. Similarly we have shown that the evolutionary mechanism of C-Frag is simply a cellular response to stress (fig. 3).

\section{Basic Research}

Because of the overwhelming heterogeneity of cell death in populations, simply monitoring the expression or activation of a protein in an extraction from a mixed population of cells is not sufficient to explain what is happening to the entire population. Many of the basic tools of molecular biology rely on profiles created from a population average. For instance, Western blots show the relative expression of a protein in a mixture of proteins derived from the lysis of a population of cells. According to our recent data, the average growth of a population is not driven by average cells from the population, but rather by highly proliferative outliers, especially in cases of high genome heterogeneity [Heng et al., 2010a, b; Abdallah et al., unpublished data]. Methods that average a given parameter, such as Western blotting, also may hide information about the processes that occur. These measurements have been used to show that induction of caspase activation following treatment by inducers of apoptosis takes place gradually. In contrast, apoptotic events occur rapidly following a prolonged period of stasis following the initial 
treatment [Goldstein et al., 2000a, b; Tyas et al., 2000]. Thus, profiling the population average is often deceiving. Furthermore, biochemical studies must be undertaken with caution as some aspects of cell death held to be almost akin to biological laws do not always hold true. For instance, apoptosis can occur without caspase involvement, and exposure of phosphatidylserine on the outer cell membrane can indicate that other forms of death have occurred [Mihalache et al., 2011]. Similarly, reliance on only morphological criteria can miss the underlying biochemical processes occurring during that death [Galluzzi et al., 2012]. In a given tumor or population, individual knowledge of the specific processes may enable a more robust induction of cell death.

Cellular heterogeneity impacts longer term results. Frequently studies of cell death focus on a short time window of either a few hours or a few days. However, studies of the results of a drug in a short time window may not be representative of the effects of heterogeneity over time. For example, if a targeted agent is applied to a heterogeneous population of cells, cells expressing the target will be eliminated over a given time period. Molecular analysis may also indicate a strong death response, but cells within the population that lack the target would be spared. Over time these few survivors repopulate the tumor, leading to resistance. In practice this is much more complex, as a drug treatment can induce adaptive genome change within the population. Thus, it is prudent to monitor the response of cells for longer periods of time, weeks, if not months.

An important aspect of cell death is the portion of the cell cycle in which the death occurs. The portion of the cell cycle in which the death occurs is often ignored but may give insight into the status of a cell. For example, in normal, healthy cells, cell cycle checkpoints exist to ensure that the cell is unstressed and important events such as DNA replication have completed. Prolonged activation of these checkpoints can lead to activation of cell death pathways. Checkpoint integrity in cancer is typically compromised, and cells often enter mitosis with extensive damage. When this occurs, a cell with sufficient damage will undergo MCD directly in mitosis via C-Frag or following an abortive mitosis via mitotic catastrophe.

\section{In the Clinic}

The behavior of individual cells within a population in response to treatment is very important. Measurements of reduction in tumor volume often do not indicate long term outcome. Initial reduction of a tumor burden may be a desirable goal, but treatment must be balanced so that the cell death that is induced is largely tumor-specific and has little off-target effects [Heng et al., 2010a; Heng, 2013b]. Furthermore, care must be taken in treatment design to avoid induction of genomic instability and subsequent increases in the evolutionary potential of a tumor. Treatments must be designed to favor the elimination of abnormal cells in diseases such as cancer to slow the disease process, while avoiding treatment induced system instability such as genome chaos.

\section{Conclusion}

Cell death heterogeneity is an important issue and is responsible for much confusion in cell death research. This is especially true in regards to the effectiveness of various cancer therapies at inducing cell death. Cell death heterogeneity often causes inconsistent outcomes, even when the same treatment is applied to the same system. While the cell death research field has had great success in identifying molecules involved in cell death, cell death is still far from being understood. Developing a deeper understanding of the multiple levels of heterogeneity in cell death is central to pushing a new era of cell death research. To accomplish this, new approaches and ideas are required that embrace complexity and eschew reliance on linear models.

Many biological questions cannot be answered with a simple yes or no, and cell death is no exception to this. Cell death is not good or bad, but rather can be both. However, in the cancer field, focus is often placed on one pathway or event in a population that is thought to be a positive marker, such as the appearance of caspase activity, which is deemed as a good result of treatment. If cells die appropriately, cell death usually occurs in a beneficial manner, and this cell death is good. If it occurs inappropriately or is incomplete, it is detrimental. Along with changes to the underlying framework of cell death research, cell death heterogeneity requires the development of new experimental and statistical tools [Heng et al., 2010a, b]. Complex statistical methods, such as multimodal analyses, are required to understand the effects of outliers. Studies must also be adapted to include longer time windows in order to appreciate phenomena such as how incomplete cell death can promote genomic diversity and long term survival while in the short term reduce tumor volume [Heng et al., 2010a]. Cell cycle information is also an important addition to future research as it can aid in the determination of the types of cell death that are occurring [Stevens et al., 2007, 2011a]. Importantly, we 
have to consider what is being measured. For instance, if caspase cascades are strongly activated but a treated tumor persists after a few weeks, are the correct metrics being assessed? We have shown that broadly defined stress is linked to cell death in general, so the focus should be on the ultimate phenotype of death, rather than on specific molecules whose interactions will diminish our ability to harness cell death in a constructive manner. We have previously introduced a similar idea to cancer research in which we use random rather than specific genome change to monitor genomic instability [Heng et al., 2006a, b]. This method has proven to be a more robust measure of tumorigenic potential than accounting for specific gene mutations or pathways. Finally, genome alteration is linked to multiple diseases [Iourov et al., 2008; Heng,
2010; Sgaramella, 2010] and plays an important role in organismal evolution [Heng et al., 2006b; Gorelick and Heng, 2011; Heng, 2013b; Horne et al., 2013b]. Therefore, cell death heterogeneity clearly requires more attention in regards to somatic and organismal evolution.

\section{Acknowledgements}

This paper is part of a series of studies entitled, 'The mechanisms of somatic cell and organismal evolution'. This work was partially supported by grants from the DOD (GW093028), the Susan G. Komen Breast Cancer Foundation, the National CFIDS Foundation, the Nancy Taylor Foundation for Chronic Diseases and SeeDNA Biotech Inc.

\section{References}

Abend M: Reasons to reconsider the significance of apoptosis for cancer therapy. Int J Radiat Biol 79:927-941 (2003).

Baek SH: When signaling kinases meet histones and histone modifiers in the nucleus. Mol Cell 42:274-284 (2011)

-Bergsmedh A, Szeles A, Henriksson M, Bratt A, Folkman MJ, et al: Horizontal transfer of oncogenes by uptake of apoptotic bodies. Proc Natl Acad Sci USA 98:6407-6411 (2001).

- Bielas JH, Loeb LA: Quantification of random genomic mutations. Nat Meth 2:285-290 (2005).

Blagosklonny MV: Apoptosis, proliferation, differentiation: in search of the order. Semin Cancer Biol 13:97-105 (2003).

-Calzone L, Tournier L, Fourquet S, Thieffry D, Zhivotovsky B, et al: Mathematical modelling of cell-fate decision in response to death receptor engagement. PLoS Comput Biol 6: e1000702 (2010).

-Castedo M, Perfettini JL, Roumie T, Andreau K, Medema R, Kroemer G: Cell death by mitotic catastrophe: a molecular definition. Oncogene 23:2825-2837 (2004a).

Castedo M, Perfettini JL, Roumier T, Valent A, Raslova H, et al: Mitotic catastrophe constitutes a special case of apoptosis whose suppression entails aneuploidy. Oncogene 23: 4362-4370 (2004b).

Chen C, Cui J, Lu H, Wang R, Zhang S, Shen P: Modeling of the role of a Bax-activation switch in the mitochondrial apoptosis decision. Biophys J 92:4304-4315 (2007).

$\checkmark$ Chen WW, Niepel M, Sorger PK: Classic and contemporary approaches to modeling biochemical reactions. Genes Dev 24:1861-1875 (2010).

-Cohen AA, Geva-Zatorsky N, Eden E, FrenkelMorgenstern M, Issaeva I, et al: Dynamic proteomics of individual cancer cells in response to a drug. Science 322:1511-1516 (2008).

\section{Darding M, Meier P: IAPS: guardians of RIPK1. Galluzzi L, Joza N, Tasdemir E, Maiuri MC, Hen- Cell Death Differ 19:58-66 (2012). gartner $\mathrm{M}$, et al: No death without life: vital}

Daugas E, Susin SA, Zamzami N, Ferri KF, Irinopoulou $\mathrm{T}$, et al: Mitochondrio-nuclear translocation of AIF in apoptosis and necrosis. FASEB J 14:729-739 (2000).

Davidoff F: Heterogeneity is not always noise: lessons from improvement. JAMA 302:25802586 (2009).

$\checkmark$ de Bruin EC, Medema JP: Apoptosis and nonapoptotic deaths in cancer development and treatment response. Cancer Treat Rev 34: 737-749 (2008).

Domingos PM, Steller H: Pathways regulating apoptosis during patterning and development. Curr Opin Genet Dev 17:294-299 (2007).

Eisenberg-Lerner A, Kimchi A: The paradox of autophagy and its implication in cancer etiology and therapy. Apoptosis 14:376-391 (2009).

Entezari M, Zakeri Z, Lockshin RA: Apoptosis in developmental processes; in: eLS (John Wiley \& Sons Ltd, Chichester 2010). http://onlinelibrary.wiley.com/doi/10.1002/9780470015902. a0022094/abstract.

Erenpreisa J, Cragg M: Mitotic death: a mechanism of survival? A review. Cancer Cell Int 1: 1 (2001).

Feng Z, Hu W, Rajagopal G, Levine AJ: The tumor suppressor p53: cancer and aging. Cell Cycle 7:842-847 (2008).

Fuentes-Prior P, Salvesen GS: The protein structures that shape caspase activity, specificity, activation and inhibition. Biochem J 384:201232 (2004).

Galluzzi L, Kroemer G: Shigella targets the mitochondrial checkpoint of programmed necrosis. Cell Host Microbe 5:107-109 (2009). functions of apoptotic effectors. Cell Death Differ 15:1113-1123 (2008a).

Galluzzi L, Morselli E, Vicencio JM, Kepp O, Joza $\mathrm{N}$, et al: Life, death and burial: multifaceted impact of autophagy. Biochem Soc Trans 36: 786-790 (2008b).

Galluzzi L, Kepp O, Kroemer G: RIP kinases initiate programmed necrosis. J Mol Cell Biol 1: 8-10 (2009).

Galluzzi L, Vitale I, Abrams JM, Alnemri ES, Baehrecke EH, et al: Molecular definitions of cell death subroutines: recommendations of the nomenclature committee on cell death 2012. Cell Death Differ 19:107-120 (2012).

Goldstein JC, Kluck RM, Green DR: A single cell analysis of apoptosis. Ordering the apoptotic phenotype. Ann NY Acad Sci 926:132-141 (2000a).

Goldstein JC, Waterhouse NJ, Juin P, Evan GI, Green DR: The coordinate release of cytochrome c during apoptosis is rapid, complete and kinetically invariant. Nat Cell Biol 2:156162 (2000b).

Gonzalez-Polo RA, Boya P, Pauleau AL, Jalil A, Larochette $\mathrm{N}$, et al: The apoptosis/autophagy paradox: autophagic vacuolization before apoptotic death. J Cell Sci 118:3091-3102 (2005).

Gorelick R, Heng HH: Sex reduces genetic variation: a multidisciplinary review. Evolution 65: 1088-1098 (2011).

-Guenebeaud C, Goldschneider D, Castets M, Guix C, Chazot G, et al: The dependence receptor UNC5H2/B triggers apoptosis via PP2A-mediated dephosphorylation of DAP kinase. Mol Cell 40:863-876 (2010).

Hendry JH, West CM: Apoptosis and mitotic cell death: their relative contributions to normaltissue and tumour radiation response. Int J Rad Biol 71:709-719 (1997). 
Heng HH: Cancer genome sequencing: the challenges ahead. Bioessays 29:783-794 (2007).

Heng HH: The genome-centric concept: resynthesis of evolutionary theory. Bioessays 31 : 512-525 (2009).

-Heng HH: Missing heritability and stochastic genome alterations. Nat Rev Genet 11:812 (2010).

Heng HH: 4D-Genomics: Genome Dynamics and Constraint in Evolution (Springer, Heidelberg 2013a).

-Heng HHQ: Bio-complexity challenging reductionism; in Sturmberg JP, Martin CM (eds): Handbook on System and Complexity in Health, pp 193-208 (Springer, Heidelberg 2013b).

-Heng HH, Stevens JB, Liu G, Bremer SW, Ye CJ: Imaging genome abnormalities in cancer research. Cell Chromosome 3:1 (2004).

-Heng HH, Bremer SW, Stevens J, Ye KJ, Miller F, et al: Cancer progression by non-clonal chromosome aberrations. J Cell Biochem 98: 1424-1435 (2006a).

-Heng HH, Stevens JB, Liu G, Bremer SW, Ye KJ, et al: Stochastic cancer progression driven by non-clonal chromosome aberrations. J Cell Physiol 208:461-472 (2006b).

-Heng HH, Bremer SW, Stevens JB, Ye KJ, Liu G, Ye CJ: Genetic and epigenetic heterogeneity in cancer: a genome-centric perspective. J Cell Physiol 220:538-547 (2009).

-Heng HH, Liu G, Stevens JB, Bremer SW, Ye KJ, Ye CJ: Genetic and epigenetic heterogeneity in cancer: the ultimate challenge for drug therapy. Curr Drug Targets 11:1304-1316 (2010a).

-Heng HH, Stevens JB, Bremer SW, Ye KJ, Liu G, Ye CJ: The evolutionary mechanism of cancer. J Cell Biochem 109:1072-1084 (2010b).

-Heng HH, Liu G, Stevens JB, Bremer SW, Ye KJ, et al: Decoding the genome beyond sequencing: the new phase of genomic research. Genomics 98:242-252 (2011a).

-Heng HH, Stevens JB, Bremer SW, Liu G, Abdallah BY, Ye CJ: Evolutionary mechanisms and diversity in cancer. Adv Cancer Res 112:217253 (2011b).

-Heppner GH: Tumor heterogeneity. Cancer Res 44:2259-2265 (1984).

-Horne SD, Stevens JB, Abdallah BY, Liu G, Bremer SW, et al: Why imatinib remains an exception of cancer research. J Cell Physiol 228:665-670 (2013a).

-Horne SD, Abdallah BY, Stevens JB, Liu G, Ye KJ, et al: Genome constraint through sexual reproduction: application of $4 \mathrm{D}$-Genomics in reproductive biology. Syst Biol Reprod Med, E-pub ahead of print (2013b).

- Iourov IY, Vorsanova SG, Yurov YB: Chromosomal mosaicism goes global. Mol Cytogenet 1:26 (2008).

Kennedy NJ, Kataoka T, Tschopp J, Budd RC: Caspase activation is required for $\mathrm{T}$ cell proliferation. J Exp Med 190:1891-1895 (1999).

Kerr JF, Wyllie AH, Currie AR: Apoptosis: a basic biological phenomenon with wide-ranging implications in tissue kinetics. Br J Cancer 26: 239-257 (1972).
Kondo S: Apoptosis by antitumor agents and other factors in relation to cell cycle checkpoints. J Radiat Res 36:56-62 (1995).

Lettre G, Hengartner MO: Developmental apoptosis in C. elegans: a complex CEDnario. Nat Rev Mol Cell Biol 7:97-108 (2006).

Luan D, Zai M, Varner JD: Computationally derived points of fragility of a human cascade are consistent with current therapeutic strategies. PLoS Comput Biol 3:e142 (2007).

-Mihalache CC, Yousefi S, Conus S, Villiger PM, Schneider EM, Simon HU: Inflammation-associated autophagy-related programmed necrotic death of human neutrophils characterized by organelle fusion events. J Immunol 186:6532-6542 (2011).

Mille F, Thibert C, Fombonne J, Rama N, Guix C, et al: The Patched dependence receptor triggers apoptosis through a DRAL-caspase-9 complex. Nat Cell Biol 11:739-746 (2009).

- Nagata S, Hanayama R, Kawane K: Autoimmunity and the clearance of dead cells. Cell 140: 619-630 (2010).

Nichols WW, Levan A: Measles associated chromosome breakage. Arch Gesamte Virusforsch 16:168-174 (1965).

Nitta M, Kobayashi O, Honda S, Hirota T, Kuninaka S, et al: Spindle checkpoint function is required for mitotic catastrophe induced by DNA-damaging agents. Oncogene 23:65486558 (2004).

- Olivier M, Petitjean A, Marcel V, Petre A, Mounawar $M$, et al: Recent advances in p53 research: an interdisciplinary perspective. Cancer Gene Ther 16:1-12 (2009).

- Park SS, Eom Y-W, Choi KS: Cdc2 and Cdk2 play critical roles in low dose doxorubicin-induced cell death through mitotic catastrophe but not in high dose doxorubicin-induced apoptosis. Biochem Biophys Res Commun 334:1014-1021 (2005).

-Park SS, Kim MA, Eom Y-W, Choi KS: Bcl-xL blocks high dose doxorubicin-induced apoptosis but not low dose doxorubicin-induced cell death through mitotic catastrophe. Biochem Biophys Res Commun 363:1044-1049 (2007).

Qu X, Yu J, Bhagat G, Furuya N, Hibshoosh H, et al: Promotion of tumorigenesis by heterozygous disruption of the beclin 1 autophagy gene. J Clin Invest 112:1809-1820 (2003).

Riedl SJ, Shi YG: Molecular mechanisms of caspase regulation during apoptosis. Nat Rev Mol Cell Biol 5:897-907 (2004).

Roninson IB, Broude EV, Chang BD: If not apoptosis, then what? Treatment-induced senescence and mitotic catastrophe in tumor cells. Drug Resist Updat 4:303-313 (2001).

Sgaramella V: Variability of our somatic (epi)genomes. Science 329:32-33 (2010).

Sigal A, Milo R, Cohen A, Geva-Zatorsky N, Klein $Y$, et al: Variability and memory of protein levels in human cells. Nature 444:643-646 (2006).

- Sit KH, Paramanantham R, Bay BH, Chan HL, Wong KP, et al: Sequestration of mitotic (Mphase) chromosomes in autophagosomes: mitotic programmed cell death in human Chang liver cells induced by an $\mathrm{OH}^{*}$ burst from vanadyl(4). Anat Rec 245:1-8 (1996).

- Spencer SL, Sorger PK: Measuring and modeling apoptosis in single cells. Cell 144:926-939 (2011).

Stephens PJ, Greenman CD, Fu B, Yang F, Bignell $\mathrm{GR}$, et al: Massive genomic rearrangement acquired in a single catastrophic event during cancer development. Cell 144:27-40 (2011).

Stevens JB, Savasan S, Liu G, Bremer SW, Atanasovski $\mathrm{M}$, et al: Characterization of drug induced chromosome fragmentation. ASHG Annual Meeting (2004). http://www.ashg. org/genetics/abstracts/abs04/f326.htm

-Stevens JB, Liu G, Bremer SW, Ye KJ, Xu W, et al: Mitotic cell death by chromosome fragmentation. Cancer Res 67:7686-7694 (2007).

- Stevens JB, Abdallah BY, Regan SM, Liu G, Bremer SW, et al: Comparison of mitotic cell death by chromosome fragmentation to premature chromosome condensation. Mol Cytogenet 3:20 (2010).

- Stevens JB, Abdallah B, Liu G, Ye CJ, Horne SD, et al: Diverse system stresses: common mechanisms of chromosome fragmentation. Cell Death Dis 2:e178 (2011a).

- Stevens JB, Abdallah BY, Horne SD, Liu G, Bremer SW, Heng HH: Genetic and epigenetic heterogeneity in cancer; in: eLS (John Wiley \& Sons Ltd, Chichester 2011b). http:// www.els.net/WileyCDA/ElsArticle/refIda0023592.html

Su H, Bidere N, Zheng L, Cubre A, Sakai K, et al: Requirement for caspase-8 in NF-kappaB activation by antigen receptor. Science 307 : 1465-1468 (2005).

- Sun SY, Yue P, Zhou JY, Wang Y, Choi Kim HR, et al: Overexpression of Bcl2 blocks TNF-related apoptosis-inducing ligand (TRAIL)-induced apoptosis in human lung cancer cells. Biochem Biophys Res Commun 280:788-797 (2001).

Tantos A, Han KH, Tompa P: Intrinsic disorder in cell signaling and gene transcription. Mol Cell Endocrinol 348:457-465 (2012).

- Taylor BF, McNeely SC, Miller HL, Lehmann GM, McCabe MJ Jr, States JC: p53 suppression of arsenite-induced mitotic catastrophe is mediated by $\mathrm{p} 21 \mathrm{CIP} 1 / \mathrm{WAF} 1$. J Pharmacol Exp Ther 318:142-151 (2006).

Tyas L, Brophy VA, Pope A, Rivett AJ, Tavare JM: Rapid caspase- 3 activation during apoptosis revealed using fluorescence-resonance energy transfer. EMBO Rep 1:266-270 (2000).

Vandenabeele P, Galluzzi L, Van den Berghe T, Kroemer G: Molecular mechanisms of necroptosis: an ordered cellular explosion. Nat Rev Mol Cell Biol 11:700-714 (2010).

Xiao AY, Wei L, Xia S, Rothman S, Yu SP: Ionic mechanism of ouabain-induced concurrent apoptosis and necrosis in individual cultured cortical neurons. J Neurosci 22:1350-1362 (2002).

Ye CJ, Liu G, Bremer SW, Heng HH: The dynamics of cancer chromosomes and genomes. Cytogenet Genome Res 118:237-246 (2007). 